\title{
Identificando necessidades de acervo de usuários de um departamento acadêmico: uma abordagem bibliométrica
}

Elias Oliveira

Professor adjunto I. Departamento de Ciência da Informação. Universidade Federal do Espírito Santo

E-mail: elias@npd.ufes.br

\section{Resumo}

A rotina de uma biblioteca universitária de médio para grande porte pode perder de vista os interesses de seus clientes: os usuários. Diante de um aporte de verba, enviam-se memorandos pedindo aos departamentos da instituição listas de itens que gostariam de ver adquiridos. Muitas vezes, os membros do departamento são pegos de surpresa e suas listas são feitas às pressas. Este trabalho apresenta uma medotologia para um sistema interativo de sugestões aos usuários para facilitar a aquisição. Tais sugestões se basearão nos indicativos de necessidades dos próprios usuários, em seus trabalhos científicos. Para a validação inicial, realizou-se experimento em um departamento universitário utilizando a metodologia proposta, na qual acertamos mais de $41 \%$ dos itens desejados pelos membros do departamento em estudo.

\section{Palavras-chave}

Monitoramento da informação; Bibliometria; Sugestões de aquisição.

\section{Identifying the user needs on library collections of an academic department: a bibliometric approach}

\begin{abstract}
A medium to large size academic library can lose sight on the users needs. At the arrival of an unexpected budget for new acquisitions, it is common to see a library's director sending memoranda to all faculty departments asking for suggestions on the new acquisitions items which they would like to have. This take, most of the time, the faculties by surprise, and their suggestions are done without a deeper reflection. This work aims to show a methodology of an interactive system for the user's suggestions in order to ease the gathering the suggestions of collection items for the acquisition. Such a methodology is based on the user's needs obtained by monitoring their own scientific work. In order to initially validate the proposed approach, we carried out an experiment on an faculty department. The result showed that we could guess more than $41 \%$ of the informational items desired by that department.
\end{abstract}

\section{Keywords}

Information monitoring; Bibliometrics; Acquisition suggestions.

\section{INTRODUÇÃO}

A produção do conhecimento em uma entidade científica é comumente medida por meio dos artigos publicados, relatórios técnico-científicos, monografias (de conclusão de curso de gradução e especialização), dissertações e teses.

No entanto, notamos que os software de muitas bibliotecas mantêm-se completamente alheios a esses valiosos instrumentos para mapear o conhecimento, como podemos ver em Côrte et alii (2002). Esse distanciamento não permite, por exemplo, que os dirigentes desses espaços se antecipem em prover as necessidades de usuários: os pesquisadores e alunos da instituição. Não raramente, os diretores de bibliotecas têm um aporte de verba e, para orientar as novas aquisições, solicitam aos membros dos departamentos da instituição que lhes enviem suas listas com os itens que gostariam de ver adquiridos. Uma atitude passiva, portanto.

Neste trabalho, além da iniciativa do usuário em expressar suas necessidades diretamente à biblioteca, independentemente do aporte de verba, queremos mostrar que os próprios trabalhos científicos desses usuários já podem indicar desejos de aquisição de novos itens por parte da biblioteca. Para isso, é necessário constante monitoramento da produção acadêmica da comunidade universitária, possibilitanto, dessa forma, que a biblioteca possa também apontar, de forma mais calculada e incisiva, itens para novas aquisições, antecipando-se à manifestação do usuário.

Este artigo está organizado da seguinte forma: na segunda seção, apresentamos algumas técnicas existentes na literatura sobre metodologias de geração e escolha de itens para aquisição ou complementação do acervo bibliotecário. Na terceira seção, apresentamos nossa proposta de um procedimento semi-automático, interativo, de auxílio a sugestões de aquisição de novos itens informacionais para o acervo. Este procedimento será baseado no monitoramento da produção científica local. Na quarta seção, apontamos algumas propostas para trabalhos futuros. Concluiremos este trabalho na quinta seção, na qual apresentaremos nossas conclusões. 


\section{AQUISIÇÃO DE ACERVO: ALGUMAS PRÁTICAS}

Na literatura existem algumas propostas de estratégias de tomada de decisão na enumeração de novos itens para inclusão ou complementação de acervo em uma biblioteca. Essas propostas vão desde a consulta direta aos membros dos departamentos de uma universidade até a utilização de um procedimento mais sofisticado de inferência (Danilowicz \& Szarski, 1981) e Kao et alii (2003). Porém, Machado \& Silva (2002) apontam que, no contexto brasileiro, pouco estudo de usuário estaria sendo feito com vistas a apoiar o desenvolvimento de coleções.

No trabalho apresentado por Kao et alii (2003), a técnica de datamining foi utilizada para a consideração dos itens a serem levados em conta no processo de aquisição de acervo. Datamining é um processo de descoberta de conhecimentos implícitos em uma grande base de dados. Esta técnica tem a capacidade de revelar relações, padrões e tendências que muitas vezes podem passar despercebidos em uma base de dados histórica, como nos aponta Kantardzic (2002).

O processo apresentado em Kao et alii (2003), batizado como Acquisition Budget Allocation Model via Data Mining (ABAMDM), consiste em mensurar, por meio dos dados históricos da circulação diária dos itens informacionais do acervo, em que nível os usuários de cada departamento se utilizam dos materiais específicos de suas áreas de atuação. Assim, essa contabilização mostrará se os membros do departamento $\mathbf{A}$, por exemplo, estão realmente utilizando os títulos de suas áreas específicas que estão disponíveis no acervo da biblioteca.

Com essa contabilização, o departamento A pode receber um peso maior do que o departamento $B$, que, sistematicamente, utiliza mais itens de outras áreas. Dessa forma, no momento de se tomar uma decisão sobre a distribuição de verba para a aquisição de novos itens, o departamento A receberia uma quantia maior da verba que o departamento B. É evidente que essa regra é altamente subjetiva, como bem observam seus autores. Entretanto, um ponto importante a se observar é que, com esse procedimento, o usuário não precisa ser consultado novamente sobre os títulos que gostaria que constassem da lista de aquisição. Todo o processo é realizado 'silenciosamente' nas bases de dados da biblioteca.

Danilowicz \& Szarski (1981), trabalhando com periódicos científicos, reúnem três classes de estratégias comumente usadas na literatura para qualificação da importância desse tipo de acervo:

1) solicitar a opinião do usuário sobre uma dada relação de periódicos (check list);

2) conferir a freqüência do acesso, em um dado tempo, ao periódico na biblioteca;

3) avaliar a quantidade de citações feitas aos artigos publicados na revista em outras mídias científicas.

Parece-nos, a partir de observações empíricas, que a opção de check list é a mais utilizada no contexto brasileiro, seja via processo manual, seja via o próprio sistema de automação de biblioteca. Isso não somente para os periódicos, mas também para outros itens informacionais do acervo da biblioteca.

Os dois outros procedimentos possibilitam uma avaliação mais precisa da real necessidade do item informacional no acervo. Ao contrário do primeiro, que depende de uma consulta direta aos usuários, são procedimentos que dependem apenas de análises feitas na base de dados da biblioteca sobre os itens já existentes no acervo.

$\mathrm{Na}$ tentativa de expressar formalmente um sistema ideal de serviço de informação, Danilowicz nos dá a seguinte definição. Seja U o conjunto dos usuários com interesse em periódicos científicos em um particular assunto ou área e J o conjunto de todas os periódicos que fornecem informação de interesse dos usuários em U, (Danilowicz \& Szarski, 1981). Dessa forma, um sistema de informação é considerado ideal se:

a) todos os artigos do conjunto J estão na conta de interesse dos usuários;

b) o sistema provê serviço de acesso para todos os usuários em $\mathrm{U}$;

c) a informação prestada aos usuários é pertinente e completa.

Em outras palavras, um sistema de informação ideal é tal que provê a todos os usuários de cada segmento do conhecimento todos os artigos de seus interesses. Como tal sistema não existe, os sistemas existentes são apenas uma aproximação do desejado. Diante disso, trabalhamos sempre com uma aproximação do que poderia ser ideal. Portanto, a proposta que apresentaremos a seguir não invalida as anteriormente apresentadas, mas as complementa com o intuito de melhorar ainda mais tal aproximação. 
Na próxima seção, discutiremos nossa proposta de um processo semi-automático de sugestões para aquisição de itens informacionais para o acervo. Esse processo baseia-se no monitoramento das referências bibliográficas elencadas pelos próprios usuários da biblioteca em seus trabalhos científicos. Tal monitoramento possibilitará uma avaliação quantitativa da importância do item requisitado e, além disso, a revelação de novos itens para a biblioteca.

\section{SUGESTÃO SEMI-AUTOMÁTICA AQUISIÇÃO VIA MONITORAMENTO}

Nossa proposta é um processo semi-automático para a escolha daqueles itens informacionais que deverão constar da lista dos desejados para a aquisição ou complementação do acervo da biblioteca. $\mathrm{O}$ processo baseia-se no fato de que a lista de referências bibliográficas dos trabalhos científicos de um pesquisador expressa o seu mapa do conhecimento (Egghe \& Rousseau, 1990).

Nós advogamos que esse mapa já diz muito do que o pesquisador necessita e, muitas das vezes, pode apontar até mesmo uma necessidade futura. Acrescenta-se a isso que, não raramente, os pesquisadores se antecipam no suprimento de suas necessidades por informação antes mesmo que o processo burocrático de uma biblioteca possa perceber tal fato. Isso ocorre quando falta na biblioteca algum item informacional necessário para o trabalho do pesquisador, obrigando-o a se antecipar, adquirindo, ele mesmo, tal item. Essa antecipação será expressa, sem dúvida, em suas referências bibliográficas.

Portanto, com base nas listas de referências bibliográficas dos trabalhos científicos dos usuários, podemos elencar itens que ainda não estejam disponíveis na biblioteca. Além disso, por meio de um processo de contagem de (re)incidência de um título nos trabalhos, é possível adquirir uma quantidade tal desses títulos que possa atender à demanda dos usuários. Queremos deixar claro que o procedimento aqui proposto não exclui outros procedimentos que já venham sendo colocados em prática na instituição.

Como um experimento-piloto, utilizaremos neste trabalho o conjunto de referências bibliográficas das dissertações de mestrado do Departamento de Informática da Universidade Federal do Espírito Santo (DI-Ufes) para aplicar nossa proposta. Nesse experimento, utilizaremos um processo manual. Entretanto, na quarta seção, apontamos possibilidades da utilização de tecnologias já existentes no mercado e que permitem automatizar completamente o método aqui proposto.

\section{Referências bibliográficas nas dissertações do DI-Ufes}

A utilização das referências bibliográficas como ferramenta para a recuperação da informação, como proposto por Zunde (1971), pode não ser a mais precisa das ferramentas, como é discutido em Simkin, por causa dos diversos motivos que levam um autor a citar uma outra obra (Simkin \& Roychowdhury, 2003; White, Wellman \& Nazer, 2004). Entretanto, a despeito dessa imprecisão, essa é uma das ferramentas que mais têm sido utilizadas pela ciência da informação para se avaliar conhecimento (Ortiz, Ortiz \& Silva, 2003).

O DI-Ufes possui um programa de mestrado iniciado em março de 1993, com a primeira dissertação defendida em dezembro de 1995. Até julho de 2003, 82 dissertações foram defendidas. Como o conjunto das referências bibliográficas das dissertações de mestrado do DI-Ufes apresenta melhor monitoramento na sua confecção que os trabalhos de alunos de graduação, nossa escolha fica restrita ao primeiro. Acreditamos, assim, que as indicações de referências bibliográficas feitas pelos pesquisadores nas dissertações apontarão uma necessidade mais fidedigna com respeito ao acervo da biblioteca.

Entretanto, do total de 82 dissertações de mestrado, tivemos acesso apenas a 46, pois as demais ainda não tinham sido disponibilizadas à biblioteca.

\section{Inferindo necessidades}

Tendo o conjunto de referências bibliográficas de um grupo de pesquisadores ao longo de alguns anos, sugerimos neste trabalho dois métodos para inferirmos suas necessidades. Uma será chamada de forma direta, e outra, de indireta.

$\mathrm{Na}$ forma direta, apenas destacamos os itens da relação de referências bibliográficas e verificamos se eles estavam inclusos ou não na lista de pedidos para novas aquisições feita pelo DI-Ufes em 2003. Já na forma indireta, utilizaremos palavras-chave a partir do título, para pesquisarmos, em bases catalográficas e portais de livrarias eletrônicas, por itens do mesmo assunto ou outros títulos similares.

Entendemos que a missão de uma biblioteca não é abrigar todos os itens bibliográficos existentes no mundo, até por questões de espaço físico. Entretanto, é preciso que ela assuma como compromisso a localização dos itens informacionais demandados para além de sua área física. Para isso, os serviços de compartilhamento de recursos entre diferentes bibliotecas devem ser constantemente 
avaliados e melhorados, veja Comut, como forma de prover ao usuário o acesso aos itens informacionais que, por algum motivo, não farão parte do acervo, a despeito de sua expressa necessidade.

Neste artigo, focalizamos apenas a aquisição de livros. No entanto, tal procedimento vale para as demais categorias: periódicos, teses e anais de conferências científicas. Nossa opção por abordar somente a categoria livro se deu em decorrência da existência de uma lista recentemente formulada pelo DI-Ufes, que foi apresentada à seção de serviço de aquisição da Biblioteca Central da mesma universidade, para subsidiar a aquisição de novos títulos que se restringiam a livros. Nessa lista, sobre a qual faremos nossas avaliações e reflexões, foram elencados 169 novos títulos a serem adquiridos.

\section{Método direto e indireto}

No conjunto das referências bibliográficas das 46 dissertações de mestrado relacionadas, encontramos um total de indicações de 375 livros, alguns repetidos. Destes, foram detectadas 26 indicações diretas, ou seja, livros que aparecem nas referências bibliográficas das dissertações e também na lista de livros pedidos pelo DI-UFES. Isso representa um índice de 15,38\% de acertos na intenção de aquisição, caso esse levantamento tivesse sido anterior à geração da lista.

Outros livros que apareceram entre as referências bibliográficas não constam diretamente da lista preparada pelo DI-UFES. Isso porque algumas dessas referências correspondem a títulos que abordam tecnologias ou metodologias mais antigas. Essas referências bibliográficas compõem as indicações indiretas, pois o título novo poderia perfeitamente cobrir o conteúdo dessas tecnologias ou metodologias mais antigas.

Para identificar tais livros, utilizamos palavras-chave dos títulos listados no conjunto de referências bibliográficas informadas no final das dissertações. Dessa forma, procuramos por outros livros existentes na lista preparada pelo DI-Ufes que abrangessem, no todo ou em parte, os assuntos descritos nas palavras-chave dos livros que foram indicados nas referências bibliográficas.

Com esse procedimento, conseguimos relacionar 54 livros como indicações indiretas, portanto $31,95 \%$ de acertos na intenção de aquisição. Entendemos que esse segundo método representa uma forma mais fraca de indicação de itens informacionais que o anterior. Entretanto, se juntarmos a lista de livros obtidos pelo método direto e indireto, observamos que $41,33 \%$ das intenções de novas aquisições já estavam cobertas. Isso permite afirmar que a biblioteca poderia antecipar as possíveis indicações de itens a serem adquiridos, o que, para o usuário, representa economia de tempo, esforço e uma demonstração de atuação proativa por parte da biblioteca.

Com esse exercício, podemos ver que o monitoramento da base bibliográfica da produção científica dos membros da comunidade pode detectar indicações de aquisição de obras para o acervo com uma certa precisão. Prática como essa já foi também sugerida por outros autores, como, por exemplo, em Coito et alii (2002).

A existência ou não dos demais 295 livros na biblioteca não foi avaliada, já que nosso intuito foi comparar a obtenção da informação explícita e implícita via referências bibliográficas de um determinado grupo da comunidade científica na Ufes. Entretanto, podemos adiantar que a aparição desses 295 livros nas referências bibliográficas também serve como uma boa fonte de informação para outras análises, como, por exemplo, aquele feito por Paula et alii (2002) para uma avaliação do uso do acervo da biblioteca.

Um ponto a salientar é que neste trabalho não entramos no mérito da quantidade de exemplares necessária na aquisição, a prioridade de um em relação a outros na lista de indicações, ou mesmo outro título substituto. Para isso, poderíamos, também, criar mecanismos de inferência por meio do material coletado nas referências bibliográficas.

\section{TRABALHOS FUTUROS}

Para este trabalho, todo o processamento sobre as listas de referências bibliográficas foi realizado manualmente. Isso porque, na Ufes, ainda não possuímos um mecanismo sistemático de coleta automatizada desses dados junto aos usuários, projeto que está em andamento e passará, em breve, por um teste-piloto no DI-Ufes. É importante notar que, para automatizar esse processo, não carecemos de uma inovação tecnológica em particular, veja Harrison (1989), pois no mercado já existem software disponíveis que dão conta desta tarefa, bastando apenas algumas complementações em sua estrutura. É o caso do EndNote, ProCite, Reference Management e, mais recentemente, o bibWord desenvolvido por Canós (2000) .

O software não é inovador, mas a efetiva operacionalização da idéia em uma biblioteca será. Apenas seria preciso fazer uma outra camada de software sobre os aplicativos anteriormente citados para a contagem dos itens informacionais. 
Nesse contexto, visualizamos dois grandes grupos de usuários: os usuários do LaTeX (Goossens et alii 1999; Lamport, 1994) - um processador de texto amplamente utilizado pela comunidade científica - e os do Microsoft Word e similares: StarOffice e OpenOffice.

Para os usuários do LaTeX, já existe um mecanismo de geração automática das referências bibliográficas que funciona de forma satisfatória e tem sido imitado por muitos outros software (Canós, 2000). Esse processo consiste na geração de uma base de dados, separada do texto principal do documento, na qual as informações de cada item bibliográfico são introduzidas: título, autoria, ano etc. Após a geração dessa base de dados, o processador de texto do LaTeX é capaz de associar, de forma automática, os pontos de identificação das fontes citadas no texto principal e gerar as estruturas dos itens da seção de referências no formato e estilo desejados pelo usuário (Lamport 1994).

Em Canós (2000), é proposto um ambiente para a geração de uma base de dados de referências bibliográficas, na qual o usuário do software Microsoft Word será capaz de introduzir as informações de cada item bibliográfico desejado para constar da seção de referências de seu trabalho científico. Uma vez criada essa base de dados, a geração das referências bibliográficas será automatizada, tal como já é feito no LaTeX.

São esses os dois procedimentos que pretendemos adotar junto aos usuários do DI-Ufes para obter, já na forma eletrônica, os itens listados na seção de referências dos trabalhos por eles produzidos. Esses itens não serão apenas livros, como feito no presente trabalho, mas também artigos, anais de congressos, dissertações e teses. Com esses itens em formato eletrônico, é possível, de forma mais rápida e fácil, analisar tais informações, por exemplo, contar quantas vezes um item aparece em diferentes trabalhos, fazendo, inclusive, uma taxonomia quanto ao tipo de trabalho em que o item é referenciado (se trabalho final de curso, dissertação, tese ou artigos científicos em geral). Isso nos dará uma indicação da importância de uma obra para aquele setor.

\section{CONCLUSÃO}

Neste trabalho, apresentamos uma proposta alternativa de sugestões para a aquisição de novos itens para o acervo de uma biblioteca. Essa proposta se baseia em análises das referências bibliográficas dos próprios membros da comunidade. Por meio dessas análises, é possível inferir a necessidade de novos itens informacionais a serem adquiridos pela biblioteca. Nossa proposta aponta um papel mais proativo para uma biblioteca, contrastando com aquele em que a biblioteca apenas solicita aos departamentos que apresentem uma lista de itens desejados para novas aquisições.

O procedimento apresentado aqui não dispõe ainda de um processo totalmente automático, mas isto será alvo de um trabalho futuro, no qual esperamos agregar modernas tecnologias já existentes no mercado para este fim. Após a automatização desse processo, esperamos também que possam ser incluídas não somente as listas de referências bibliográficas dos pesquisadores, mas também a bibliografia que acompanha o programa de disciplinas oferecidas nos cursos de graduação e pósgraduação, como foi proposto por Eleutério \& Prati (2002).

Um problema que enfrentamos na realização deste trabalho foi a falta de alguns serviços especializados de acesso on-line em sites de bibliotecas e livrarias brasileiras - acesso de forma automática às suas bases de dados via linguagem de programação, sem intervenção humana, portanto. Por causa disso, só foi possível fazer acessos on-line automatizados para nossos experimentos usando o site da empresa www.amazon.com, que oferece tal serviço de consulta.

Artigo recebido em 20-11-2003 e aceito para publicação em 24-04-2004. 
Identificando necessidades de acervo de usuários de um departamento acadêmico: uma abordagem bibliométrica

\section{REFERENCIAS}

COMUT. Programa de Comutação Bibliográfica (Brasília, DF). Disponível em: <http://www.ibict.br>. Acesso em: 14 nov. 2003.

CANÓS, J. H. A bibliography manager for microsoft word. ACM Crossroads Student Magazine, New York, n. 64, Summer 2000. Disponível em: <http://www.acm.org/crossroads/xrds6-4/ bibword.html>. Acesso em: 14 nov. 2003

COITO, M. I. et al. Avaliação do uso da coleção de periódicos por docentes e alunos da Faculdade de Ciências Farmacêuticas - UNESP. In: SEMINÁRIO NACIONAL DE BIBLIOTECAS UNIVERSITÁRIAS, 12., 2002, Recife, PE. Anais... Recife : UFPE, 2002. 1 CD-ROM.

CÔRTE, A. R. et al. Avaliação de softwares para bibliotecas e arquivos. 2. ed. São Paulo : Polis, 2002

DANILOWICZ, C.; SZARSKI, H. Selection of scientific journals based on the data obtained from an information service system. Information Processing EO Management, v. 17, n. 1, p. 13-19, 1981.

EGGHE, L.; ROUSSEAU, R. Introduction to informetrics: quantitative methods in library, documentation and information science. Amsterdan : Elsevier Science, 1990.

ELEUTÉRIO; PRATI, S. C. Diagnóstico da acessibilidade à bibliografia recomendada nos cursos de graduação das áreas de saúde e biológicas na Universidade de São Paulo - Campus de São Paulo In: SEMINÁRIO NACIONAL DE BIBLIOTECAS UNIVERSITÁRIAS, 12., 2002, Recife. Anais... Recife : UFPE, 2002. 1 CD-ROM.

GOOSSENS, M. et al. The LaTeX web companion: integrating TeX, HTML and XML. Massachuestts: Addison-Wesley, 1999.

HARRISON, M. A.; MUNSON, E. V. On integrated bibliography processing. Electronic Publishing-Origination, Dissemination and Design, v. 2, n. 4, p. 193-209, 1989. Disponível em: <http://citeseer.nj.nec.com/article/harrison95integrated.html>. Acesso em: 14 nov. 2003.

KANTARDZIC, M. Data mining : concepts, models, methods and algorithms. New York : IEEE, 2002.

$\mathrm{KAO}$, S. et al. Decision support for the academic library acquisition budget allocation via circulation database mining. Information Processing Ė Management, v. 39, n. 1, p. 133-147, 2003

LAMPORT, L. LaTeX: a document preparations system. 2. ed. Massachuestts : Addison- Wesley, 1994.

MACHADO, R. N.; Silva, Z. P. Desenvolvimento de coleç̃es: uma análise a partir dos anais SNBUs realizados na década de 90. In: SEMINÁRIO NACIONAL DE BIBLIOTECAS UNIVERSITÁRIAS, 12, 2002, Recife, PE. Anais... Recife : UFPE, 2002. 1 CD-ROM.

ORTIZ, L. C.; ORTIZ, W. A.; SILVA, S. L. Ferramentas alternativas para monitoramento e mapeamento automatizado do conhecimento. Ciência da Informação, Brasília, v. 31, n. 3, p. 66-76, 2003.

PAUlA, T. M. B. et al. Avaliação das citações em dissertações e teses do deparamento de ciência política da FFLCH/USP defendidas no ano de 2000: um estudo de uso. In: SEMINÁRIO NACIONAL DE BIBLIOTECAS UNIVERSITÁRIAS, 12., 2002, Recife, PE. Anais... Recife : UFPE, 2002. 1 CD-ROM.

SIMKIN, M. V.; ROYCHOWDHURY, V. P. Copied citations create renowned papers? Disponível em: <http://arxiv.org/abs/cond-mat/ 0305150>. Acesso em: 14 nov. 2003.

WHITE, H.; WELLMAN, B.; NAZER, N. Does citation reflect social structure? Journal of the American Society for Information Science and Technology, v. 55, n. 2, p. 111-126, 2004.

ZUNDE, P. Structural models of complex information sources. Information Storage and Retrieval, v. 7, n. 1, p. 1-18, 1971. 